\title{
RAPID GEOMETRY DEFINITION FOR MULTIDISCIPLINARY DESIGN AND ANALYSIS OF AN AIRCRAFT
}

\author{
Jacek MIELOSZYK ${ }^{1}$, Tomasz GOETZENDORF-GRABOWSKI ${ }^{2}$, Dawid MIESZALSKI ${ }^{3}$ \\ ${ }_{1,2}$ Aircraft Design, Power and Aeronautical Engineering, Nowowiejska 24, 00-665 Warsaw, Poland \\ ${ }^{3}$ MSP company, Poligonowa 1/81, 04-051 Warsaw, Poland

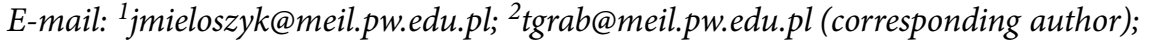 \\ 3dawid.mieszalski@gmail.com
}

Received 17 September 2014; accepted 25 March 2016
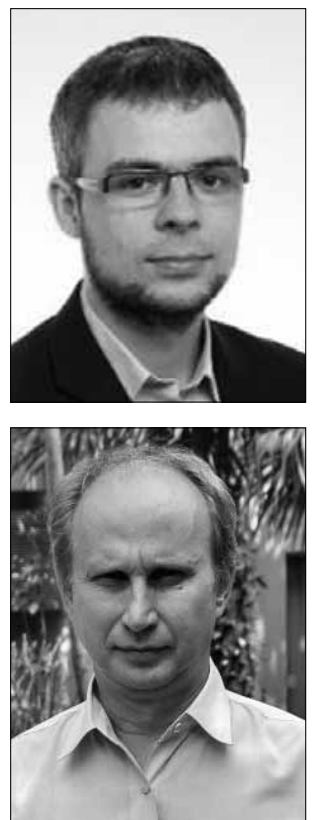

Jacek MIELOSZYK, defended PhD on The Faculty of Power and Aeronautical Engineering at Warsaw University of Technology. Member of PSAA - Polish Society of Aeronautics and Astronautics. Representative of Pegasus - international network of aerospace universities. Currently conducts research activities in Aircraft Design Department of The Faculty of Power and Aeronautical Engineering at Warsaw University of Technology.

Interested in multidisciplinary methods of aircraft design and optimization. He focuses his work on the development of new tools for aircraft design, automation of the aircraft design processes and development of algorithms for numerical optimization.

Tomasz GOETZENDORF-GRABOWSKI, Faculty of Power and Aeronautical Engineering, Warsaw University of Technology

Associate professor in Aircraft Design Department in Warsaw University of Technology. Education: MSc (Engineering) - 1985 in aeronautics, MSc (Engineering) - 1987 in applied mechanics, PhD - 1994 in flight mechanics; D.Sc - 2014 in mechanics. Affiliations and functions: 1986-1988 - Light Aircraft Company PZL -Okecie - Flight simulator division, from 1989 - Warsaw University of Technology - Lecturer of Flight Mechanics, Optimization in Aircraft Design, 1996-2005 - ETC-PZL Aerospace Industries Ltd - software division - flight dynamics and simulation expert; from 2011 - vice director for general affairs of Institute of Aeronautics and Applied Mechanics.

Member of PSAA - Polish Society of Aeronautics and Astronautics, ICAS Programme Committee member

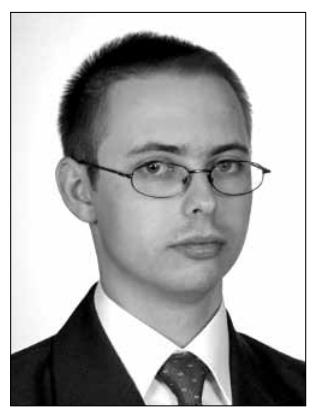

Dawid MIESZALSKI, MSP Marcin Szender company

Unmanned aerial vehicles flight control and navigation systems designer at MSP company. Co-designer of mini class reconnaissance and surveillance fixed-wing UAV.

$H e$ is interested in user-friendly and reliable UAV ground control station system and UAVs operator interaction with software during missions

BSc (2006) and MSc (2008) on The Faculty of Power and Aeronautical Engineering at Warsaw University of Technology.

Abstract. Conceptual and preliminary design level of aircraft design is searching for an easy, flexible and efficient way of computational geometry definition. Accelerating the process of geometry definition is the basic step for acceleration of all computations. It also enables optimization, where changes of numerical model are made automatically according to the optimization algorithms. The geometry definition has to be robust, free from errors and stay feasible.

Keywords: geometry definition, multidisciplinary, design, analysis. 


\section{Introduction}

A number of efforts are taken worldwide, by well known institutions to realize this claim. The European project SimSAC (Rizzi 2011) that began in 2006 concentrated on the enhancement of conceptual design and early preliminary design processes by introducing the integrated environment CEASIOM (www.ceasiom.com) for multidisciplinary analysis, with special attention to aircraft stability and control characteristics. The software package is capable of multidisciplinary analysis on different levels of fidelity, including: geometry and mesh generation, mass model definition, FEM analysis, aerodynamic analysis and stability and control analysis.

A new approach to software generation also has to guarantee it's flexibility and robustness in future development and maintenance. In Virginia Polytechnic, the basis of special common language Aircraft Design Markup Language (ADML) (Deshpande et al. 2013) for aircraft design was set. The basic concept utilizes the main concepts and features of the common markup language XML. Markup coding languages are easily extensible, allowing for great flexibility of future development and maintenance of tools for aircraft design. They do not provide ready data structures, but rather flexible rules to define the structures, utilizing concept of classes, encapsulation, polymorphism and so on. The language is human readable, allowing for interaction in substandard cases directly on the code to create innovative aircraft configurations and solutions such as a joinwing configuration. These languages also offer standard procedures of data consistency checking to avoid basic data errors. The difference between ADML and XML is that the basic structures, common in aerospace computations field of science, are already provided. This allows for fast definition of computational cases and efficient management of data. Similar efforts were taken by the DLR institute to define a platform of common information sharing between different faculties in the institute. The faculties have detailed knowledge about the science disciplines they are involved in and a basic one about other aerospace disciplines, but all have to share the same data when designing an aircraft. The DLR created the Common Parametric Aircraft Configuration Schema language CPACS (Nagel et al. 2012, Rizzi et al. 2012, http:// software.dlr.de/p/cpacs/home/) also based on the main XML language features.

The main effort in defining the ADML language was taken bottom-up for defining powerful constructs for functions and abstract mathematical objects, with unconventional aircraft configurations in mind, while the CPACS concentrated on the top-down way for an entire aircraft definition to single data objects like point lists, and currently it can only handle traditional aircraft designs.

At Warsaw University of Technology, department of Aircraft Design, we established a concept of tools for Multidisciplinary Aircraft Design and Optimization, MADO (Goetzendorf-Grabowski et al. 2012), by joining the main stream concepts of the SimSAC project, the ADML and CPACS languages. The MADO package contains a group of programs: OptoM for multidisciplinary optimization, SDSA for nonlinear dynamic stability and control analysis and Panukl (http://www.meil. pw.edu.pl/add/ADD/Teaching/Software/PANUKL) for pre-processing, aerodynamic analysis, data export for SDSA (Goetzendorf-Grabowski et al. 2011) and CalculiX/Abaqus FEM software (http://www.calculix.de/) and post-processing. The core assumptions of the concept are:

- user friendly interface with errors warning;

- less complexity with maximum efficiency of computational cases definition;

- new concepts of advanced geometry definition for fast geometry definition;

- easy and automated data exchange for multidisciplinary analysis and optimization;

- enabling creation of innovative aircraft configurations;

- flexibility of the future development and maintenance of the software tools.

\section{Geometry definition environment}

One of the most pleasant things to do in the MADO package is carry out efficient and error free computational geometry definition in the Graphical User Interface (GUI) called the Ms2editor (Fig. 1). There is also a possibility to define the geometry in plain text, but this is not recommended since the GUI suggests options to choose and provides error checking. The tool is very powerful and flexible in use.

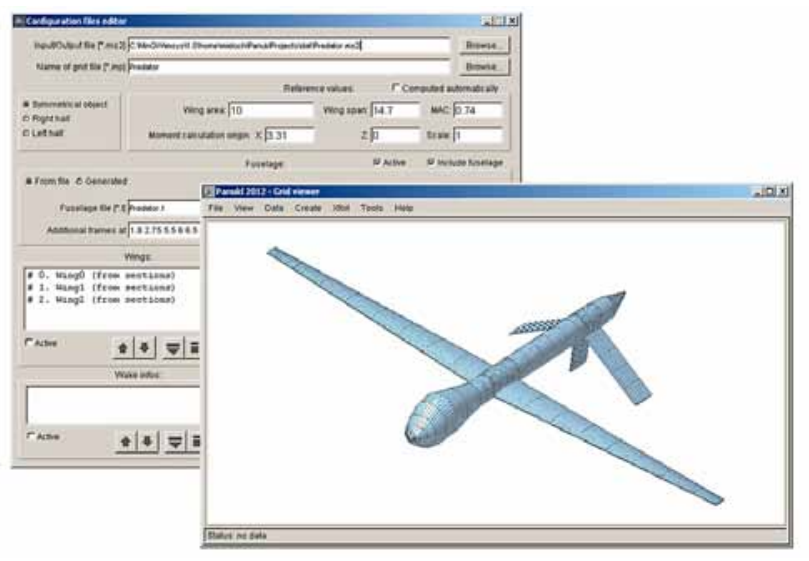

Fig. 1. The GUI of the MS2editor (Panukl pre-processor) 


\section{New concepts in geometry definition}

We introduce a new concept of building the geometry through modular wing definition. Previously, wings were built using separately defined sections containing airfoil coordinates properly placed in a three dimensional space. This way of building a wing geometry is easy when dealing with fairly simple geometry, for example a trapeze wing plan form, but becomes very inefficient when wing geometry contains more complicated three dimensional forms like smooth winglets with arcs or joined wings. Building geometry using the modular wing concept is similar to building with different blocks (line, arc, etc.) added to one another. The advantages are that a group of cross sections is defined at once, with less parameters to set and a guarantee that the geometry connection is satisfied, also, a smooth geometry with a tangency condition of arcs and linear parts of the wing can be achieved in this way.

To visualize it better, an example of a definition of wing with winglet is presented (Fig. 2). The basic geometry consists of a horizontal linear part, an arc at the joint of the main wing and winglet, which is the vertical linear part. The arc module automatically computes a tangency constrain to the main wing module. The setting of the same angles for arc ending and winglet module will provide tangency for the arc-winglet joint (Fig. 2a). A few adjustments can still be made. To add a fuselage, the first panel of the planar module of the wing can be made bigger to fit the fuselage. Nonlinear paneling distribution is improved both chord wise and span wise. If the wing should have minimum induced drag, the option "Optimize for minimum induced drag" enables it (Munk law (Jones 1979) is used to calculate optimum chord distribution, assuming constant design lift coefficient and defined reference area), as shown in Fig. $2 b$. The airfoil's position in the chord wise direction is set in the end (Fig. 2c). The definition of the wing geometry requires to set only a few parameters. It is easy, fast and flexible to build wings with modules.

Definition of the fuselage is also very easy with the help of the Ms2editor's widgets. The concept of the definition of the fuselage is shown in Fig. 3a. The shape of a fuselage is defined by the main contours (up contour, side contour and down contour). Paneling along the longitudinal axis of the fuselage is set by the projection of

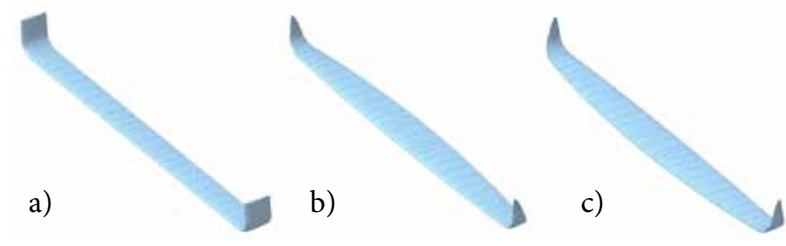

Fig. 2. Modular wing geometry with winglets panels from the super ellipse (Mason 2006) on the spine. Finally, convexity distribution of the fuselage upper and lower quarters along the longitudinal axis is defined. The convexity idea is illustrated in Fig. 3b. The grid with a defined wing and fuselage is shown in Fig. 4. The different convexity on the upper and lower parts of the fuselage is emphasized.

It is also possible to define nacelles with only a few parameters (Fig. 5). The profiles for the nacelle can be inverted, which will create an accelerating or decelerating effect. The convexity factors of the nacelle make it easy to create nacelles with very different forms, as in Fig. 6.

The MADO environment for geometry creation offers even more unique and innovative concepts. Examples of generated grids containing sophisticated geometry are shown in Fig. 7, Fig. 8, and Fig. 9.

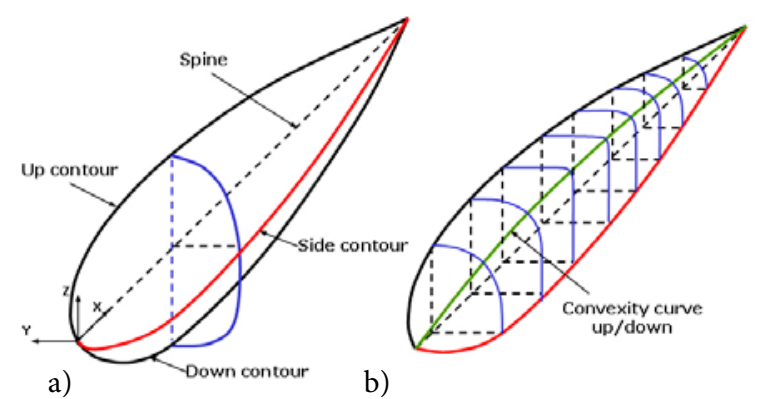

Fig. 3. Concept of fuselage definition

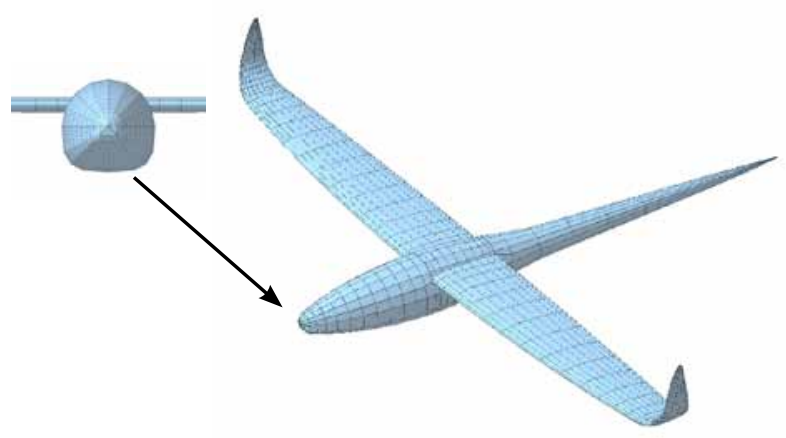

Fig. 4. Grid with a different convexity of the upper and lower fuselage quarters
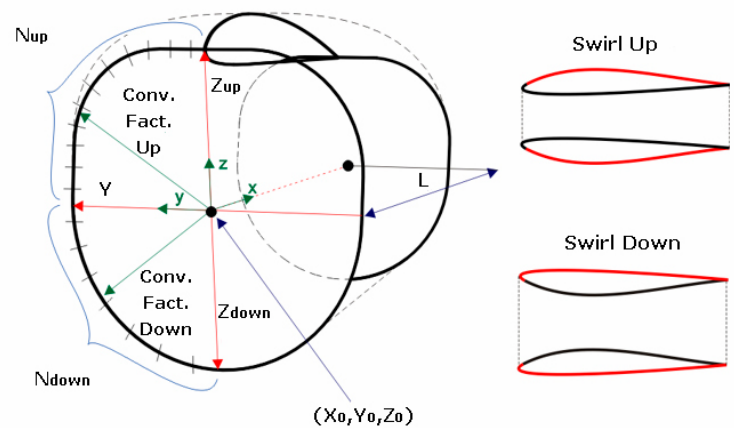

Fig. 5. Nacelle parameters 

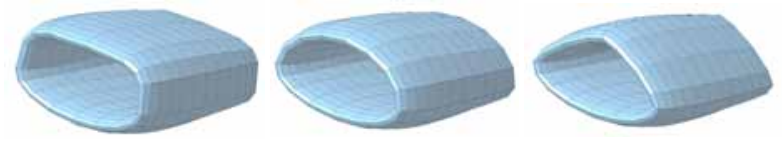

Fig. 6. Examples of nacelles with different "Conv. Fact."

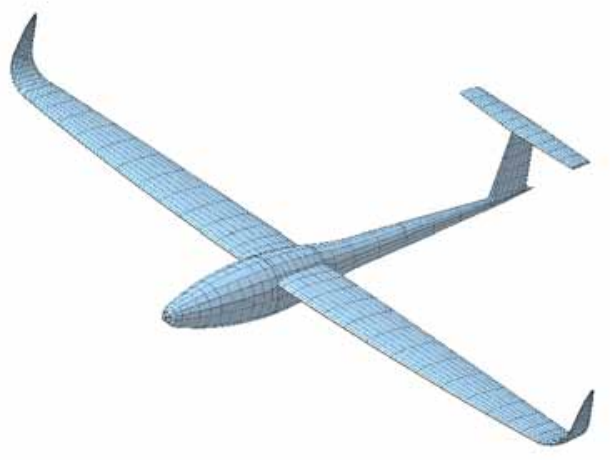

Fig. 7. Advanced glider grid.

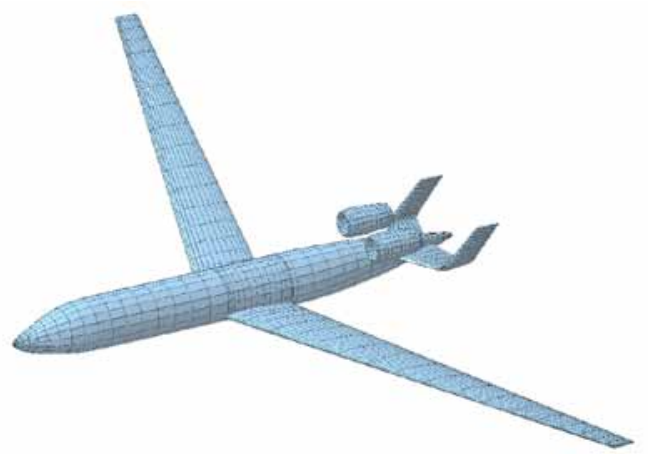

Fig. 8. Grid of innovative transport aircraft.

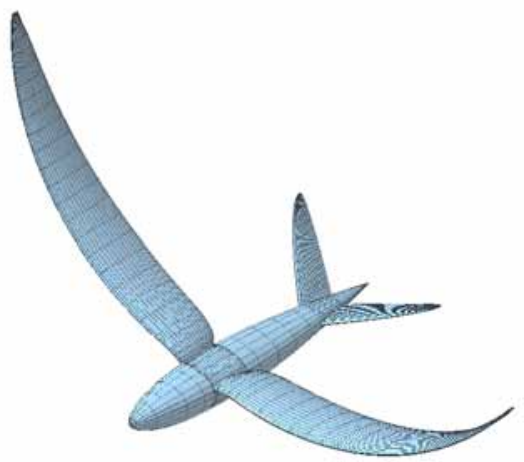

Fig. 9. Fantastic airplane benchmark for geometry generation tools.

\section{Geometry for optimization}

Tools for grid definition permit an easy preparation of associative geometry for optimization. Geometry parameters can be changed manually in the Ms2editor GUI, or changed by numerical optimization variables. An example of a difficult-to-define geometry used for optimization of a novel aircraft in a joinwing configuration is

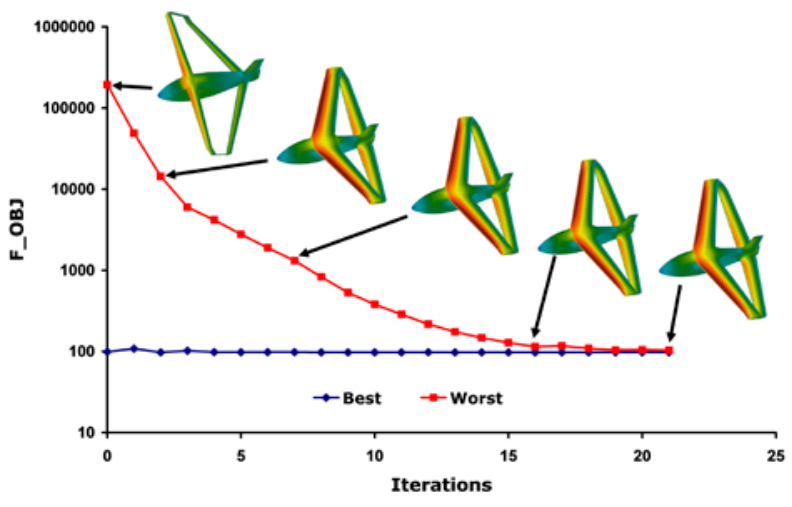

Fig. 10. Optimization of joinwing aircraft geometry.

illustrated in Fig. 10. For the optimization, the Monte Carlo algorithm was used. The objective function was aircraft aerodynamic efficiency with a constrain on the equality of gravity and lift forces. In this optimization case, fifteen geometry optimization variables were used. This was the preliminary stage of optimization, as work on the project is still ongoing.

\section{XML file format exchange}

To satisfy the condition of exchanging geometry data between software tools, the XML standard was used. It allows for data structuring, connection with existing standards (i.e. the CPACS) and, consequentially, for easy extension of software functionality, development and maintenance. Storing the data definition of an aircraft in a single file prevents information loss during data exchange. The textual file format of the XML makes it human readable and easy to modify by the user. Moreover, due to the structural form of the XML documents, different applications can use different parts of the whole schema without knowledge about the rest of the schema. Besides this, it is possible to check document consistency without analyzing particular data by using an appropriate XSD schema. This approach saves developers' time.

\section{Conclusions}

The package of tools for rapid geometry definition for multidisciplinary design and analysis of an aircraft contains many innovative concepts. The software provides an easy and error-free way of geometry definition. The concept proves to be efficient, fast and flexible.

\section{Acknowledgments}

This work was partially supported by The National Center for Research and Development through the grant PBS1/A6/14/2012. 


\section{References}

Calculix - A Free Software Three-Dimensional Structural Finite Element Program. Available from Internet: http:// www.calculix.de/

Common Parametric Aircraft Configuration Schema (CPACS ). Available from Internet: http://software.dlr.de/p/cpacs/ home/

Computerised Environment for Aircraft Synthesis and Integrated Optimisation Methods. Available from Internet: www.ceasiom.com

Deshpande, S.; Watson, L. T.; Love, N. J.; Canfield, R. A.; Kolonay, R. M. 2013. ADML: Aircraft Design Markup Language for Multidisciplinary Aircraft Design and Analysis, TR-13-07, Virginia Polytechnic Institute \& State University.

Goetzendorf-Grabowski, T., Mieszalski, D.; Marcinkiewicz, E. 2011. Stability analysis using SDSA tool, Progress in Aerospace Science, Vol 47, pp.636-646.

Goetzendorf-Grabowski, T.; Mieloszyk, J.; Mieszalski, D. 2012. MADO - software package for high order multidiscyplinary aircraft design and optimization, in Proceedings of 28th International Congress of the Aeronautical Sciences, 2012, Melbourne.

Jones, R. T. 1979. Classical Aerodynamic Theory. NASA Reference Publication 1050.

Mason, W. H. 2006. Configuration Aerodynamics. Virginia Tech, Blacksburg.

Nagel, B.; Böhnke, D.; Gollnick, V.; Schmollgruber, P.; Rizzi, A.; La Rocca, G.; Alonso, J .J. 2012. Communication in aircraft design: can we establish a common language?, in Proceedings of 28th International Congress of the Aeronautical Sciences, 2012, Melbourne,

Rizzi, A. 2011. Modeling and simulating aircraft stability and control. The SimSAC project, Progress in Aerospace Science, Vol 47, pp.573-588.

Rizzi, A.; Zhang, M.; Nagel, B.; Boehnke, D.; Saquet, P. 2012. Towards a unified framework using CPACS for geometry management in aircraft design, in 50th AIAA Aerospace Sciences Meeting Including the New Horizons Forum and Aerospace Exposition, 09-12 January 2012, Nashville, USA.

PANUKL potential solver, Software package, Warsaw University of Technology. Available from Internet: http://www.meil. pw.edu.pl/add/ADD/Teaching/Software/PANUKL 